\title{
Antioxidant activities of natural phenolic compounds from Acacia confusa bark
}

\author{
Yu-Tang Tung a , Jyh-Horng Wu b, Yueh-Hsiung Kuo ${ }^{\text {c }}$, Shang-Tzen Chang a,* \\ ${ }^{a}$ School of Forestry and Resource Conservation, National Taiwan University, Taipei 106, Taiwan \\ ${ }^{\mathrm{b}}$ Department of Forestry, National Chung-Hsing University, Taichung 402, Taiwan \\ ${ }^{\mathrm{c}}$ Department of Chemistry, National Taiwan University, Taipei 106, Taiwan
}

Received 27 January 2006; received in revised form 19 April 2006; accepted 21 April 2006

Available online 12 June 2006

\begin{abstract}
The present study showed that the ethanolic extracts from the bark of Acacia confusa exhibited a strong antioxidant activity. Among all the fractions from ethanolic extracts of bark, the EtOAc soluble fraction exhibited the best antioxidant performance. Furthermore, following by CC and HPLC, 11 pure phenolic compounds were isolated and identified from the ethanolic extracts.

(C) 2006 Elsevier Ltd. All rights reserved.
\end{abstract}

Keywords: Acacia confusa; Bark; Antioxidant activity; Free radical; Phenolic compounds

\section{Introduction}

Free radical reactions, especially with participation of oxidative radicals, have been shown to be involved in many biological processes that cause damage to lipids, proteins, membranes and nucleic acids, thus giving rise to a variety of diseases (Datta et al., 2000). Reactive oxygen species (ROS) have been recognized as playing an important role in the initiation and/or progression of various diseases such as atherosclerosis, inflammatory injury, cancer and cardiovascular disease (Halliwell, 1997). Thus, recent studies have investigated the potential of plant products to serve as antioxidants against various diseases induced by free radicals (Hou et al., 2003). Additionally, it has been determined that the antioxidant effect of plant products is mainly due to phenolic compounds, such as flavonoids, phenolic acids, tannins and phenolic diterpenes (Chung et al., 1998; Pietta, 2000). There have been numerous studies on the biological activities of phenolics, which are potent antioxidants and free radical scavengers (Kahkonen et al., 1999; Sugihara et al., 1999).

\footnotetext{
* Corresponding author. Tel.: +88623366 4626; fax: +886223654520. E-mail address: peter@ntu.edu.tw (S.-T. Chang).
}

Acacia confusa is traditionally used as a medicinal plant in Taiwan. An aqueous extract of $A$. confusa leaves has been shown to have wound healing and anti-blood-stasis properties (Kan, 1978). The crude extracts of $A$. confusa bark not only contain a wide variety of phenolic compounds but also show an excellent antioxidant activity (Chang et al., 2001). Therefore, this plant might be a good candidate for further development as a nutraceutical or for its antioxidant remedies. However, the potential health benefits of the bark extracts of $A$. confusa have not been studied in detail to date. In this study, CC and HPLC were employed to separate and purify the $A$. confusa bark extracts; and their antioxidant activities were further evaluated by 1,1-dipheny1-2-picrylhydrazyl (DPPH) free radical scavenging assay. In addition, the specific antioxidant compounds were identified by MS and NMR.

\section{Methods}

\subsection{Chemicals}

1,1-Dipheny1-2-picrylhydrazyl (DPPH), Folin-Ciocalteu reagent, 3,4,5-trihydroxybenzoic acid, benzoic acid, and quercetin were all purchased from Sigma Chemical Co. 
(St. Louis, MO). All other unlabelled chemicals and reagents were of analytical grade.

\subsection{Preparation of plant extracts}

The bark of $A$. confusa was sampled from the experimental forest of National Taiwan University in Nan-Tou county. The species was identified by Sheng-You Lu of the Taiwan Forestry Research Institute and a voucher specimen (AC001) was deposited at School of Forestry and Resource Conservation, National Taiwan University. The dried samples $(7.2 \mathrm{~kg})$ were cut into small pieces and soaked in $70 \%$ ethanol at ambient temperature for 7 days. The extracts were decanted, filtered under vacuum, concentrated in a rotary evaporator and then lyophilized. The resulting powder extracts were then extracted successively with $n$-hexane $(74.5 \mathrm{~g}), \quad$ EtOAc $(333.5 \mathrm{~g}), n$-butanol $(564.0 \mathrm{~kg})$, and water $(465.6 \mathrm{~g})$. After removing the solvents used for the successive extractions, four soluble fractions were obtained. The EtOAc soluble fraction was divided into 20 subfractions (EA1-EA20) by chromatography with a silica gel 60 (Merck, Darmstadt, Germany) column eluted with EtOAc/ $n$-hexane (gradient elution was performed by changing from $2 / 98$ to 100/0). Eleven compounds were isolated and purified from the EA12, EA13, EA14, and EA17 subfractions by HPLC with a Si-60 column (Mobile phase: EtOAc/ $n$-hexane $=30 / 70$; Flow rate: $4 \mathrm{ml} / \mathrm{min}$ ).

\subsection{DPPH (1,1-Diphenyl-2-picrylhydrazyl) free radical scavenging assay}

The DPPH free radical scavenging activity of $A$. confusa bark extracts was examined according to the method reported by Chang et al. (2001). First, $50 \mu 1$ of test sample in methanol (final concentrations were $1,5,10,50 \mu \mathrm{g} / \mathrm{ml}$, respectively) were mixed with $450 \mu \mathrm{l}$ of $50 \mathrm{mM}$ Tris- $\mathrm{HCl}$ buffer (pH 7.4) and $1500 \mu \mathrm{l}$ of $0.1 \mathrm{mM}$ DPPH-ethanol solution. After $30 \mathrm{~min}$ of incubation at ambient temperature, the reduction of the DPPH free radical was measured by reading the absorbance at $517 \mathrm{~nm}$. Quercetin was used as a positive control. The inhibition ratio (percent) was calculated according to the following equation: $\%$ inhibition $=[$ (absorbance of control-absorbance of sample)/absorbance of control] $\times 100$.

\subsection{Determination of total phenolics}

Total phenolic contents were determined according to the Folin-Ciocalteu method (Kujala et al., 2000), using gallic acid as a standard. The test samples were dissolved in $5 \mathrm{ml}$ of methanol/water (50/50). The sample solution $(500 \mu 1)$ was mixed with $500 \mu \mathrm{l}$ of $1 \mathrm{~N}$ Folin-Ciocalteu reagent. The mixture was allowed to stand for $5 \mathrm{~min}$, which was followed by the addition of $1 \mathrm{ml}$ of $20 \% \mathrm{Na}_{2} \mathrm{CO}_{3}$. After 10 min of incubation at ambient temperature, the mixture was centrifuged for $8 \mathrm{~min}(12,000 \mathrm{~g})$, and the absorbance of the supernatant was measured at $730 \mathrm{~nm}$. The total pheno- lic contents were expressed as gallic acid equivalents (GAE) in milligrams per gram sample.

\section{Results and discussion}

\subsection{Free radical scavenging activity of bark extracts of A. confusa}

DPPH is one of the compounds that has a proton free radical with a characteristic absorption, which decreases significantly on exposure to proton radical scavengers (Yamaguchi et al., 1998). It is well accepted that the DPPH radical scavenging by antioxidants is attributable to their hydrogen donating ability (Chen and Ho, 1995). In this study, the free radical scavenging activity of its derived fractions of $A$. confusa was assessed by DPPH assay. As shown in Table 1, except for the $n$-hexane fraction, all the test samples showed a significant inhibitory activity against the DPPH radical. Among them, EtOAc and $\mathrm{BuOH}$ fractions were the best. The complete inhibition of DPPH radical by test samples was observed at a range of $5-10 \mu \mathrm{g} / \mathrm{ml}$. In addition, the $\mathrm{IC}_{50}$ values (the concentration required to inhibit radical formation by $50 \%$ ) of the crude extract, $n$ hexane fraction, EtOAc fraction, $\mathrm{BuOH}$ fraction, and water fraction were $3.5,>100,3.2,3.6$, and $4.4 \mu \mathrm{g} / \mathrm{ml}$, respectively. The $\mathrm{IC}_{50}$ value of a well-known antioxidant compound used as a reference in this study, quercetin, is $1.6 \mu \mathrm{g} / \mathrm{ml}$. Compared with pycnogenol from the bark of the French maritime pine (Pinus maritima), a commercial product, the $\mathrm{IC}_{50}$ value is approximately $5 \mu \mathrm{g} / \mathrm{ml}$ (Packer et al., 1999). Thus, the bark extracts of $A$. confusa might be a good candidate to be developed into a commercial product.

Plant phenolics, in general, are highly effective free radical scavengers and antioxidants. The content of total phenolics in each fraction was determined spectrometrically according to the Folin-Ciocateu method and calculated as gallic acid equivalents (GAE). Accordingly, the total phenolic content of the EtOAc fraction $(505.9 \mathrm{mg} / \mathrm{g})$ was significantly higher than that of the $\mathrm{BuOH}$ fraction $(323.3 \mathrm{mg} / \mathrm{g})$, the water fraction $(401.8 \mathrm{mg} / \mathrm{g})$, and the $n$ hexane fraction $(0.0 \mathrm{mg} / \mathrm{g})$. These results indicated that the free radical scavenging activity of ethanolic extracts of $A$. confusa bark could be effectively enriched in the EtOAc fraction. Thus, the EtOAc fraction was further investigated for its phytochemical characteristics and in vitro antioxidant activity.

Table 1

$\mathrm{IC}_{50}$ values of ethanolic extracts from the bark of $A$. confusa against DPPH free radical

\begin{tabular}{ll}
\hline Specimens & $\mu \mathrm{g} / \mathrm{ml}$ \\
\hline Crude extract & 3.5 \\
$n$-Hexane fraction & $>100$ \\
EtOAc fraction & 3.2 \\
BuOH fraction & 3.6 \\
Water fraction & 4.4 \\
Quercetin & 1.6 \\
\hline
\end{tabular}




\subsection{Isolation and identification of phenolic antioxidants from the bark of $A$. confusa}

The phenolic antioxidants of EtOAc fraction were purified and identified by using HPLC, MS and 1D and 2D NMR spectrometry. Eleven phenolic compounds (1-11) were isolated and identified as 3,4-dihydroxybenzoic acid (1) (Flamini et al., 2001) (4.3 g); 3-hydroxy-4-methoxybenzoic acid (2) (2.6 g); 3,4-dihydroxybenzoic acid methyl ester (3) (7.3 g); 3,4-dihydroxybenzoic acid ethyl ester (4) (Baderschneider and Winterhalter, 2001) (5.7g); 4-hydroxybenzoic acid ethyl ester (5) (Owen et al., 2003) (0.9 g); 4-hydroxybenzoic acid (6) (Lo et al., 2002) (0.5 g); 4hydroxy-3,5-dimethoxybenzoic acid (7) (Hideyuki et al., 1980) (2.3 g); 4-hydroxy-3,5-dimethoxybenzoic acid ethyl ester (8) (4.3 g); 3,4-dihydroxy-trans-cinnamic acid ethyl ester (9) (Sugiura et al., 1989) (1.9 g); 3,4-dihydroxy-transcinnamic acid pentyl ester (10) (Naito et al., 1991) (0.7 g); and 4-hydroxy-3-methoxybenzoic acid (11) (Lee et al., 2004) (9.7 g). The ${ }^{1} \mathrm{H},{ }^{13} \mathrm{C}$ NMR, and MS spectral data of compounds 1-11 were in good agreement with the published values.

\subsection{Antioxidant activity of phenolic compounds}

Presently, information on the distribution of phenolic acids within plants is very limited. Compounds $\mathbf{1 , 2}, \mathbf{4}, \mathbf{6}$, and $\mathbf{1 1}$ were five major phenolic compounds in the EtOAc fraction from $A$. confusa bark. To examine the structureantioxidant activity relationships, two other analogues including benzoic acid and 3,4,5-trihydroxybenzoic acid were selected and their antioxidant activity compared. The scavenging effects of these compounds are shown in Fig. 1 Their scavenging activity of the DPPH free radical decreased in the following order: 3,4,5-trihydroxybenzoic acid =3,4-dihydroxybenzoic acid=3,4-dihydroxybenzoic

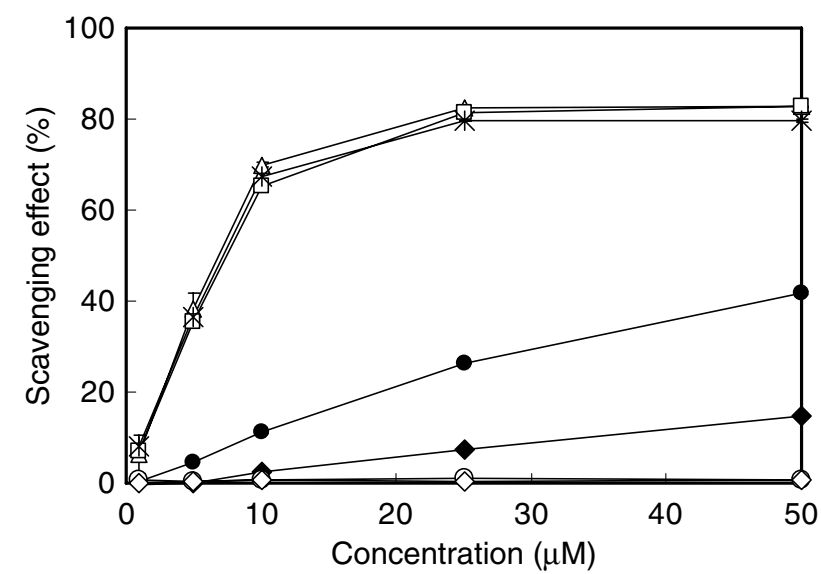

Fig. 1. Free-radical scavenging activity of various benzoic acids measured by the DPPH assay: $(\triangle)$ 3,4,5-Trihydroxybenzoic acid, $(\square)$ 3,4-Dihydroxybenzoic acid, $(\times)$ 3,4-Dihydroxybenzoic acid ethyl ester, $(\bullet)$ 4Hydroxy-3-methoxybenzoic acid, ( ) 3-Hydroxy-4-methoxybenzoic acid, $(\bigcirc)$ 4-Hydroxybenzoic acid, $(\diamond)$ Benzoic acid. Results are mean \pm SD $(n=3)$. acid ethyl ester $>4$-hydroxy-3-methoxybenzoic acid $>3$ hydroxy-4-methoxybenzoic acid $>4$-hydroxybenzoic acid $=$ benzoic acid with all values significantly different at $p<0.05$. This sequence indicated that the free radical scavenging activity of the test compounds was due to their hydrogen-donating ability. These results also suggested that catechol skeletons, such as 3,4,5-trihydroxybenzoic acid, 3,4-dihydroxybenzoic acid and 3,4-dihydroxybenzoic acid ethyl ester, had better antioxidant activity.

\section{Conclusions}

In this study, it was demonstrated for the first time that the ethanolic extracts from $A$. confusa bark possessed an excellent antioxidant activity based on the DPPH free radical assay. Some of the compounds isolated from $A$. confusa bark exhibited the strongest radical scavenging activity. Future studies should focus on the employment of modern medical chemical techniques to modify the structures of certain purified plant ingredients into better agents with high efficacy and activity. In addition, in vivo pharmacological studies also should be made.

\section{Acknowledgements}

We thank the National Science Council for the financial support (NSC94-2313-B-002-045) and the experimental forest of National Taiwan University for providing A. confusa materials, as well as Ms. Shou-Ling Huang (Department of Chemistry, National Taiwan University) for NMR spectral analyses.

\section{References}

Baderschneider, B., Winterhalter, P., 2001. Isolation and characterization of novel benzoates, cinnamates, flavonoids, and lignans from riesling wine and screening for antioxidant activity. J. Agric. Food Chem. 49, 2788-2798.

Chang, S.-T., Wu, J.-H., Wang, S.-Y., Kang, P.-L., Yang, N.-S., Shyur, L.-F., 2001. Antioxidant activity of extracts from Acacia confusa bark and heartwood. J. Agric. Food Chem. 49, 3420-3424.

Chen, C.-W., Ho, C.-T., 1995. Antioxidant properties of polyphenols extracted from green and black tea. J. Food Lipids 2, 35-46.

Chung, K.-T., Wong, T.-Y., Huang, Y.-W., Lin, Y., 1998. Tannins and human health: a review. Crit. Rev. Food Sci. Nutr. 38, 421-464.

Datta, K., Sinha, S., Chattopadhyay, P., 2000. Reactive oxygen species in health and disease. Natl. Med. J. India 13, 304-310.

Flamini, G., Antognoli, E., Morelli, I., 2001. Two flavonoids and other compounds from the aerial parts of Centaurea bracteata from Italy. Phytochemistry 57, 559-564.

Halliwell, B., 1997. Antioxidants and human diseases: a general introduction. Nutr. Rev. 55, S44-S52.

Hideyuki, C., Shigenori, T., Masao, I., 1980. Phenolic germination inhibitors in the seed balls of Red Beet (Beta vulgaris L. var. rubra). Agric. Biol. Chem. 44, 205-208.

Hou, W.C., Lin, R.D., Cheng, K.T., Hung, Y.T., Cho, C.H., Chen, C.H., Hwang, S.Y., Lee, M.H., 2003. Free radical-scavenging activity of Taiwanese native plants. Phytomedicine 10, 170-175.

Kahkonen, M.P., Hopia, A.I., Vuorela, H.J., Rauha, J.P., Pihlaja, K., Kujala, T.S., Heinonen, M., 1999. Antioxidant activity of plant extracts containing phenolic compounds. J. Agric. Food Chem. 47, 3954-3962. 
Kan, W.S., 1978. Leguminosae. In: Manual of Medicinal Plants in Taiwan. In: Kan, W.-S. (Ed.), National Research Institute of Chinese Medicine, 2. Taipei, Taiwan, pp. 239-240.

Kujala, T.S., Loponen, J.M., Klika, K.D., Pihlaja, K., 2000. Phenolics and betacyanins in red beetroot (Beta vulgaris) root: distribution and effect of cold storage on the content of total phenolics and three individual compounds. J. Agric. Food Chem. 48, 5338-5342.

Lee, C.K., Lu, C.K., Kuo, Y.H., Chen, J.Z., Sun, G.Z., 2004. New prenylated flavones from the roots of Ficus beecheyana. J. Chin. Chem. Soc. $51,437-442$

Lo, W.L., Chang, F.R., Hsieh, T.J., Wu, Y.C., 2002. The constituents of Euchresta formosana. J. Chin. Chem. Soc. 49, 421-426.

Naito, Y., Sugiura, M., Yamaura, Y., Fukaya, C., Yokoyama, K., Nakagawa, Y., Ikeda, T., Senda, M., Fujita, T., 1991. Quantitative structureactivity relationships of catechol derivatives inhibiting 5-lipoxygenase. Chem. Pharm. Bull. 39, 1736-1745.

Owen, C., James, K., Sampson, L., Ahmed, S., 2003. Synthesis and biochemical evaluation of some novel benzoic acid based esters as potential inhibitors of oestrone sulphatase. J. Pharm. Pharmacol. 55, 85-93.

Packer, L., Rimbach, G., Virgili, F., 1999. Antioxidant activity and biologic properties of a procyanidin-rich extract from pine (Pinus maritima) bark, pycnogenol. Free Radic. Bio. Med. 27, 704-724.

Pietta, P.G., 2000. Flavonoids as antioxidants. J. Nat. Prod. 63, 1035-1042.

Sugihara, N., Arakawa, T., Ohnishi, M., Furuno, K., 1999. Anti- and prooxidative effects of flavonoids on metal induced lipid hydroperoxidedependent lipid peroxidation in cultured hepatocytes located with $\alpha$-linolenic acid. Free Radic. Biol. Med. 27, 1313-1323.

Sugiura, M., Naito, Y., Yamaura, Y., Fukaya, C., Yokoyama, K., 1989. Inhibitory activities and inhibition specificities of caffeic acid derivatives and related compounds toward 5-lipoxygenase. Chem. Pharm. Bull. 4, 1039-1043.

Yamaguchi, T., Takamura, H., Matoba, T., Terao, J., 1998. HPLC method for evaluation of the free radical-scavenging activity of foods by using 1,1,-diphenyl-2-picrylhydrazyl. Biosci. Biotechnol. Biochem. 62, 12011204. 